\title{
Polar Liquid Crystal Elastomers Cross Linked Far from Thermodynamic Phase Transitions: Dislocation Loops in Smectic Clusters
}

\author{
Yusril Yusuf, ${ }^{1}$ Shohei Yamaguchi, ${ }^{2}$ Shinya Kawano, ${ }^{2}$ Hirotaka Okabe, ${ }^{2}$ Simon Krause, ${ }^{3}$ \\ Heino Finkelmann, ${ }^{3}$ P. E. Cladis, ${ }^{4}$ and Shoichi Kai ${ }^{2,5}$ \\ ${ }^{1}$ Physics Department, Faculty of Mathematics and Natural Sciences, Gadjah Mada University, Yogyakarta 55281, Indonesia \\ ${ }^{2}$ Department of Applied Quantum Physics and Nuclear Engineering, Graduate School of Engineering, Kyushu University, \\ Fukuoka 819-0395, Japan \\ ${ }^{3}$ Makromolekulare Chemie, Universität Freiburg, 79104 Freiburg, Germany \\ ${ }^{4}$ Advanced Liquid Crystal Technologies, P.O. Box 1314, Summit, NJ 07902, USA \\ ${ }^{5}$ Department of Life Engineering, Graduate School of Systems Life Sciences, Kyushu University, Fukuoka 819-0395, Japan
}

Correspondence should be addressed to Yusril Yusuf; yusril@ugm.ac.id

Received 5 February 2013; Accepted 16 June 2013

Academic Editor: Michael C. Tringides

Copyright (c) 2013 Yusril Yusuf et al. This is an open access article distributed under the Creative Commons Attribution License, which permits unrestricted use, distribution, and reproduction in any medium, provided the original work is properly cited.

\begin{abstract}
Nematic networks with three different concentrations of polar and nonpolar mesogens and the same concentration of a novel crosslinking agent give rise to unusual liquid single crystal elastomers (LSCEs) that are transparent monodomain nematic networks with smectic clusters. The largest spontaneous length change is observed in the sample with $70 \mathrm{~mol} \%$ of the polar mesogen which also has the highest glass transition temperature and smectic clusters with a slowly increasing but nearly constant layer spacing on cooling from $90^{\circ} \mathrm{C}$ to $25^{\circ} \mathrm{C}$. X-ray scattering intensity from smectic clusters with layer spacings that monotonically increase on cooling first increases to a maximum at $T^{*} \sim 60^{\circ} \mathrm{C}$ corresponding to clusters of about 30 layers. Below $T^{*}$, the scattering intensity decreases as the number of layers in a cluster decreases. To account for this surprising nonlinear behavior that correlates with nonlinear features of the networks' macroscopic spontaneous shape change and birefringence, a model is proposed where dislocations form in the layers at $T^{*}$. Below $T^{*}$, more dislocations form to break down the layer structure. The possibility of dislocation formation at $T^{*}$ independent of mesogenic concentrations is attributed to a conformational change in the crosslinker which is present at the same concentration in the three LSCEs.
\end{abstract}

\section{Introduction}

Liquid crystal states of matter have long range orientational order characterized by a director, $\mathbf{n}$, which can be oriented anywhere in three-dimensional space. Liquid crystals are an example of a continuous medium where $\mathbf{n}$ is defined everywhere in the sample except at line defects. Nevertheless, it is interesting to probe differences between low-molecularweight nematic liquid crystals, familiar as the light switch in flat panel displays, and liquid crystal elastomers resulting when a low-molecular-weight liquid crystal is polymerized and cross linked in one process to make a polydomain liquid crystal elastomer (LCE) [1-5]. Particulary as, following polymerization and cross linking, monodomain LCEs where $\mathbf{n}$ is locked in the sample can be prepared and studied $[2,4,6,7]$. The resulting liquid single crystal elastomers (LSCEs) change their lengths significantly in the vicinity of the cross-linking temperature, $T_{c}$, which can be, but need not be [8], within $5 \mathrm{~K}$ of the clearing temperature of the liquid crystal phase cross linked in the elastomer.

The notion that cross-linking temperature, $T_{c}$, is a relevant thermodynamic parameter is a novel concept in the field of liquid crystal elastomers. While being not obvious for LSCEs cross linked in the vicinity of the nematic-isotropic 

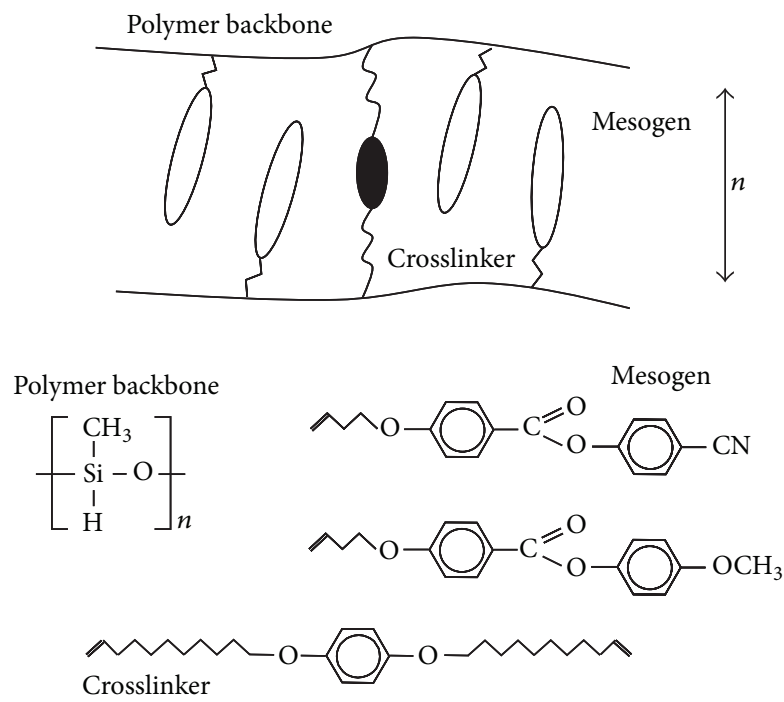

FIGURE 1: Top: sketch of one layer of polar mesogens and its crosslinker in a side-chain liquid crystal elastomer (LCE) with layer spacing larger than one pendant cyano mesogen. Below are the chemical structures of the compounds used to synthesize LSCE films $300 \mu \mathrm{m}$ thick and several $\mathrm{cm}^{2}$ area, with $7 \mathrm{~mol} \%$ crosslinker and 50, 70 , and $100 \mathrm{~mol} \%$ of the polar cyano mesogens, respectively, CN50, CN70, and CN100. Fully extended, molecular lengths of the two mesogens are $\ell \sim 20.5-21 \AA$. Including the siloxane as a chain extender adds another $\sim 2 \AA$ to each monomer. The layer spacings are shown in Figure 2.

phase transition temperature, $T_{\mathrm{NI}}$, of the LC moieties, their importance became clearer while studying LCEs cross linked far from $T_{\mathrm{NI}}[8,9]$ and thus is well supported by experiments and observations of the elastomers synthesized by the Finkelmann method, and this leads to a natural explanation for the unusual transparency of the monodomain elastomers discussed here. It also opens the door onto new experiments and questions concerning the physics of LC elastomers.

Elastomers have no liquid phases and no critical fluctuations, only relaxation dynamics. Thus, the symmetry of nematic liquid crystalline elastomers is not the same as it is in low molecular-weight liquid crystals. As a result, LCEs of the type shown in Figure 1 are important to learn how polymerization and cross-linking influence liquid crystal order. This is particularly the case for the polar cyano mesogen in Figure 1 where liquid crystal phases appear in the elastomer that did not exist before cross-linking [10].

To learn more about the correlation between liquid crystalline order at the microscopic level and the macroscopic properties of spontaneous length change measured by $\lambda \| \mathbf{n}$ and degree of orientational order measured by birefringence, $\Delta n$, in the context of previous studies for bifunctional and trifunctional cross-linked LSCEs [7, 11-15], we synthesized LSCEs with the two different mesogenic groups and the novel cross-linker in Figure 1 with layer spacings in Figure 2 and then studied their microscopic structure by synchrotron radiation (Figure 3).

An unusual feature of the elastomers in Figure 3 is that they are more than $80 \%$ transparent at $20^{\circ} \mathrm{C}$ near the

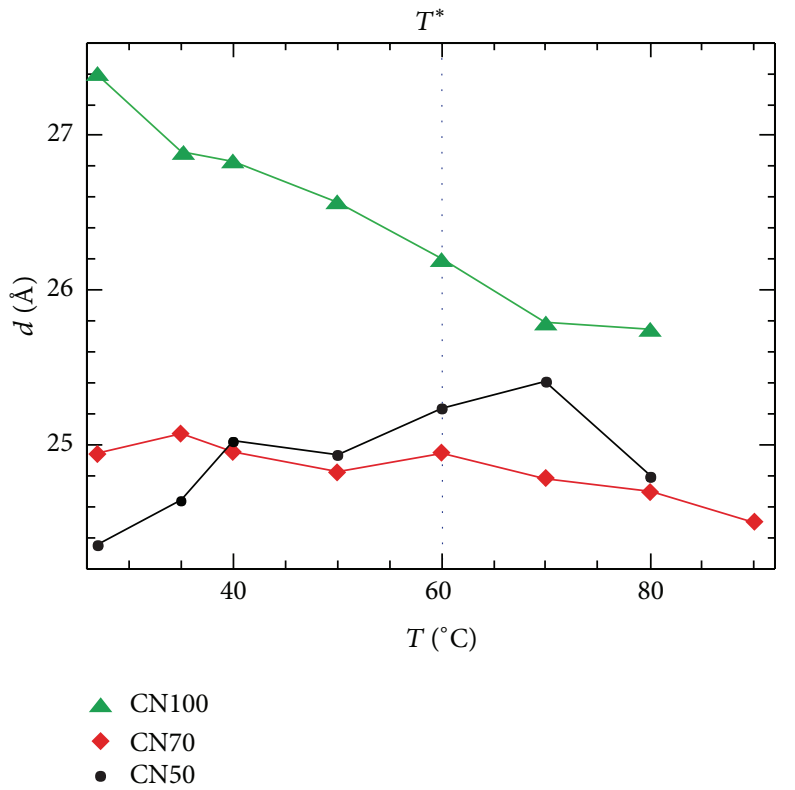

FIGURE 2: Layer spacing, $d(\AA)$ versus temperature, $T\left({ }^{\circ} \mathrm{C}\right)$, for smectic clusters in $\bullet$ CN50, $\bullet$ CN70, and $\Delta \mathrm{CN100}$. The data points are connected by lines.

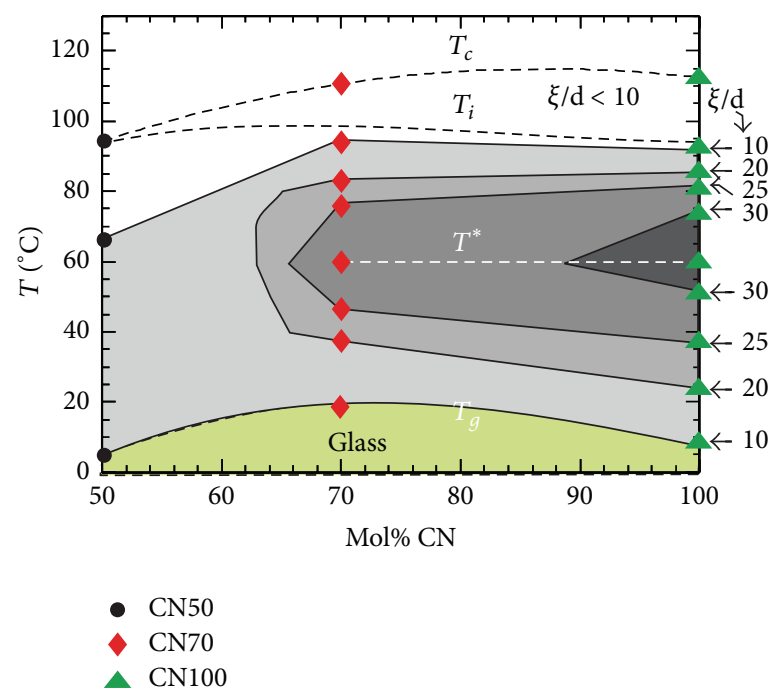

FIGURE 3: An overview of the nematic networks of $\bullet$ CN50, $\bullet$ CN70, and $\Delta \mathrm{CN100}$. The solid curves are lines of constant number of smectic layers correlated in a cluster, $\xi / d$ (shown to the right of the figure), with $\xi$, the layer correlation length, and $d$, the layer spacing (Figure 2), measured by synchrotron radiation. $\xi / d$ is maximum ( 28.3 layers for CN70, and 31.5 layers for $\mathrm{CN100}$ ) on the dashed line $T^{*}=60^{\circ} \mathrm{C}$ between CN70 and CN100 compared to about 10 layers at $T_{i}$ where smectic clusters first appear. $T_{c}$ is the cross-linking temperature. $T_{g}$ is the glass transition temperature. The maximum number of correlated layers in CN50 is 12 at $\sim 40^{\circ} \mathrm{C}$ and is only about 2 at $T_{i}$ and 10 at $T_{g}$. The dashed curve for $T_{i}$ is deduced from measurements of spontaneous shape change, $\lambda(T)$, measured in the optical microscope, discussed in Section 2.3. $T_{g}$ is estimated from the $\lambda(T)$ 's in Figure 4. 


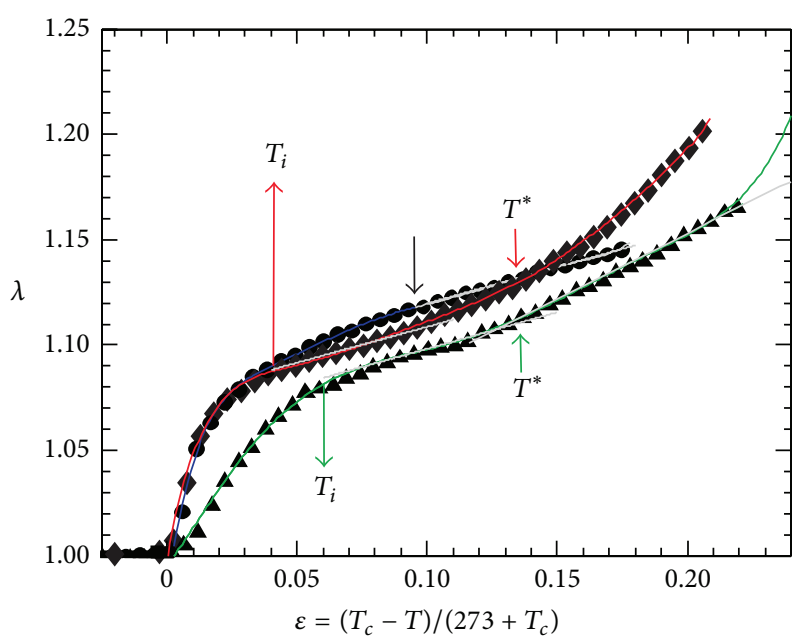

CN100
CN70
- CN50

FIgURE 4: Length change data, $\lambda$, for $\boldsymbol{\Delta} \mathrm{CN100} \bullet \mathrm{CN70}$, and $\bullet \mathrm{CN} 50$ versus $\varepsilon$, with $T_{c}=94.2^{\circ} \mathrm{C}(\mathrm{CN} 50) ; 110.6^{\circ} \mathrm{C}(\mathrm{CN} 70)$, and $112.6^{\circ} \mathrm{C}$ (CN100) plotted as symbols. The $\varepsilon \geq 0\left(T \lesssim T_{c}\right)$ solid curves superposing that the data are 6 th-order polynomial fits. To compare with the phase plot in Figure 3, arrows show that $T^{*}=60^{\circ} \mathrm{C}$ for the three CN compositions and $T_{i}$ for $\mathrm{CN} 70\left(95^{\circ} \mathrm{C}\right)$ and $\mathrm{CN} 100\left(90^{\circ} \mathrm{C}\right)$. For CN50, $T_{i} \sim T_{c}$. Data for $T>T^{*}$ is linear for CN100 up to $\varepsilon \sim 0.2$ and nonlinear for CN70 (see text for details).

glass transition temperature, $T_{g}$, taking $100 \%$ transparency at $130^{\circ} \mathrm{C}$, well above $T_{c}$, a kind of clearing temperature above which there are no more changes in $\Delta n$ and $\lambda$.

Up to now, all the nematic elastomers we studied were translucent rather than transparent. Transparency means that the $2 \mathrm{D}$ network in a smectic layer is more ordered in these LSCEs. What would be a turbid reentrant nematic in low-molecular-weight liquid crystals is interpreted for these elastomers as resulting from dislocation formation leaving behind a nematic network with enhanced orientational order with fewer and/or smaller smectic clusters.

The values for $T_{g}$ in Figure 3 are estimates based on an extrapolation of the fits, to be discussed in the next section, for Figure 4 to $T=0^{\circ} \mathrm{C}$. They are about $10 \mathrm{~K}$ higher than those obtained by Kundler and Finkelmann [5] for similar elastomers that did not exhibit reentrant behavior. More research is needed to understand better the interplay between orientational order and a glassy state below $T_{g}$, an interesting but still open question [16]. The data in these experiments suggests enhancement, rather than saturation, of orientational order as $T \rightarrow T_{g}$, consistent with the appearance of smectic clusters after polymerization and cross-linking of the cyano mesogen [10].

1.1. Low-Molecular-Weight Liquid Crystals. In low-molecular weight liquid crystals, the thermodynamic state called nematic is liquid in all three spatial dimensions so that light travels at the same speed in the two directions perpendicular to $\mathbf{n}$ as it also does in the state called smectic $A$ which is layered along the $\mathbf{n}$ axis and liquid in the layers, similar to soap bubbles. The optic axis in low-molecular-weight nematic liquid crystals is unique. It is an example of an uniaxial liquid crystal designated $D_{\infty h}$ in group theory, where $D$ refers to the $\mathbf{n}$ axis which can be oriented anywhere in space (hence the subscript $\infty$ ) with mirror planes, designated by the subscript $h$.

The nematic X-ray diffraction pattern, an interference process requiring information from at least two rays, shows only a diffuse wide angle ring corresponding to molecular dimensions and, because of fluctuations in $\mathbf{n}$, little to no scattering along $\mathbf{n}$. In addition to the long-range orientational order of the nematic phase, smectic A liquid crystals have layers with spacing typically a molecular length, 20-35 $\AA$, compared to about $\sim 5 \AA$, corresponding to a molecular width. As a result, in the small angle limit, X-ray diffraction by smectic layers in low-molecular-weight liquid crystals are nearly resolution-limited peaks along $\mathbf{n}$ [17]; that is, its line shape or structure factor, the Fourier transform of a free energy, is a sharply peaked Lorentzian function [18], with diffuse scattering on a wide ring $\perp \mathbf{n}$ for a $2 \mathrm{D}$ isotropic liquid.

The nematic-to-smectic A phase transition has been studied in great detail in low-molecular-weight liquid crystals; see, for example, Chapter 6 in [19]. It is a first-order phase transition because fluctuations in the smectic A-order parameter opens a gap in the spectrum of orientational fluctuations of $\mathbf{n}$ [20]. When the gap is small, X-ray scattering in the small angle limit grows from essentially nothing in the nematic phase far below the transition temperature to the smectic $A$ phase, $T_{\mathrm{NA}}$, with a Lorentzian structure factor of characteristic width usually denoted by $1 / \xi$, that narrows continuously on approaching the transition temperature to the smectic A phase; that is, $1 / \xi \rightarrow 0$ as $T \rightarrow T_{\mathrm{NA}}$. For $1 \mathrm{D}$ translational order (smectic A), while $\xi$ can be quite large, it can never be infinite because of the Landau-Peierls instability [19].

In low-molecular-weight liquid crystals, there are many smectic A phases differentiated by the difference between the molecular length of the compounds in the layers and the layer spacing exhibited by the smectic. When the layer spacing is between one and two molecular lengths, the smectic phase is designated $\mathrm{A}_{d}$, where $d$ here stands for dimer. In cyano mesogens, dimerization is believed to be important for the occurrence of reentrant nematic phases because dimers do not pack efficiently into layers perpendicular to the long axis of the dimer. Decreasing the free volume around a dimer by either increasing pressure or decreasing temperature can easily destabilize dimers in favor of a more dense uniaxial nematic or a biaxial smectic $C$ phase with layers tilted relative to $\mathbf{n}[10,19,21]$.

1.2. Liquid Crystal Elastomers. The layered structure perpendicular to the orientation axis, $\mathbf{n}$, is a $2 \mathrm{D}$ network in elastomers, not the 2D isotropic liquid; it is in low-molecularweight liquid crystals. Elastomers with a locked-in orientation axis can never be isotropic $[2,4,6,7]$, that is, have no access to the full symmetry group of rotations and reflections 
known as $O(3)$. As a result, there is exponential decay to some constant value above $T_{c}$ (the foot in [7]) but no jump at the first-order phase transition at $T_{c}$. We suggest that the nematic phase in elastomers could be $D_{n h}$ nematic with the director an $n$-fold rotation axis with this $n$ an integer, $n=2,3,4,5$, $6 \ldots$

Adding layers $\perp \mathbf{n}$, the director, to a $D_{n h}$ nematic leads to a smectic phase with also $n$-fold rotational symmetry in the layers rather than the continuous rotational symmetry of the smectic A phase in low-molecular-weight liquid crystals that is sometimes modeled as a $2 \mathrm{D}$ disordered network. The $n$-fold rotational symmetry in the plane of the layers accounts better for the unusual transparency of these elastomers than a disordered $2 \mathrm{D}$ network. Its wide angle X-ray diffraction pattern would be a modulated diffuse ring with an integral number of more intense but still diffuse regions every $2 \pi / n$. While being difficult to observe modulated diffuse wide angle rings in the so-called homeotropic geometry $[7,11-15]$ where the sample plane is perpendicular to $\mathbf{n}$, such an experiment would provide valuable information, currently missing, on LSCE structure.

Nematic phases with discrete rotational symmetry around $\mathbf{n}$ would be more transparent and could conserve volume in the sense used to account for spontaneous length change. For example, shrinkage parallel to $\mathbf{n}$ is exactly compensated by expansion in the two directions $\perp \mathbf{n}$. It is easy to show that such a constant volume LSCE is biaxial [22].

A thermomechanical effect at a constant-volume, firstorder phase transition was early recognized as a candidate for thermally driven artificial muscles [23, 24]. Following Warner and Kutter, we call this effect spontaneous shape change [25] and leave the important outstanding question of LCE symmetry, evident in LSCEs where $T_{c}$ is far from the clearing temperature of the mesogens cross linked in the elastomer, for separate discussions [22]. More thoughts and data are needed to speak more about LSCE symmetry.

\section{Experiment and Analysis}

2.1. Samples Involving Cyanophenyl-Alkoxybenzoates. The mesogens are a nonpolar nematic with a methoxy $\left(\mathrm{OCH}_{3}\right)$ end group and a polar nematic with a cyano $(\mathrm{CN})$ end group [10]. Aliphatic chains at the other end of each mesogen attach to the siloxane polymer backbone cross linked with $7 \mathrm{~mol} \%$ of the flexible bifunctional crosslinker (Figure 1). The samples are 50,70, and $100 \mathrm{~mol} \%$ of CN mesogens, respectively, CN50, $\mathrm{CN70}$, and CN100 in Figure 3 with layer spacings shown in Figure 2.

Smectic phases with a $\mathrm{CN}$-end group favor a combination of partially overlapped molecular pairs (fully extended dimer $\sim 27 \AA$ ) and single molecules (fully extended $\sim 21 \AA$ ). The dimer fraction, extracted from layer spacing measurements, is sensitive to temperature. With decreasing temperature, the layer spacing increases signaling an increase in dimer formation (Figure 2).

Smectic phases with a nonpolar end group have a layer spacing typically a molecular length $(\sim 20.5 \AA)$. On cooling
CN50, the slightly larger than monomolecular layer spacing first increases a little then at $T^{*}$, it decreases a little. CN50 behaves as a nematic network with smectic clusters down to $T_{g}$ (Figures 2 and 3 ).

In CN70, there is a competition between these two types of temperature dependence resulting in the layer spacing being nearly independent of temperature (Figure 2).

From what is known for the hexyl and larger members of the homologous series (4-cyanophenyl $4^{\prime}$-n-alkoxybenzoate) for this polar mesogen [10], we estimate for $n=4$, the transition to the isotropic liquid $T_{\mathrm{NI}} \simeq 87^{\circ} \mathrm{C}$ and to coexistence of smectic A with an unidentified state called $K 2$ for $T_{\mathrm{NA}^{\dagger}} \lesssim 65^{\circ} \mathrm{C}$. However, on extended heating in the isotropic liquid state, $K 2$, melts leading to a nematic with a higher transition temperature to the isotropic phase and a smectic A phase on cooling [10]. The shortest homologue showing a smectic A phase is the octyl-alkoxybenzoate and the reentrant nematic was only observed in its mixtures with shorter or less polar compounds.

As the cross-linking temperature is so high, it appears that enantiomeric properties of the lower temperature phases of cyanophenyl compounds play an important role in the synthesis of LC elastomers. In the case of cyanophenyls, the LCE has mesogenic properties that do not exist in neat 4cyanophenyl $4^{\prime}$-butyl-alkoxybenzoate including clusters of a more ordered dimerized smectic phase with layers that break down at lower temperatures. In addition, in the elastomer $T_{c}$ is enhanced by $\sim 25 \mathrm{~K}$ and $T_{g}$ is relatively high.

This raises the question would a better understanding of how cross-linking temperature affects enantiomeric properties of mesogens in the synthesis of LC elastomers be relevant to fields such as pharmacology and medicine where enantiomorphism is known to be important?

Kundler and Finkelmann did not observe reentrant-type behavior in a similar but more complex LSCE with two cross-linking agents where the fraction of $\mathrm{CN}$ mesogens was less than $50 \mathrm{~mol} \%$ [5] and the glass transition temperatures, $T_{g} \approx 0^{\circ} \mathrm{C}$. With more than $50 \mathrm{~mol} \% \mathrm{CN}$-mesogen and only one cross linking agent, the LSCEs studied here are nematic networks with smectic $\mathrm{A}$ clusters ranging from 10 to 30 layers with reentrant behavior in CN70 and CN100 but not in CN50 (Figure 3). Indeed, CN70 and CN100 are the first LSCEs to show reentranttype behavior.

2.2. LSCE Samples with Polar and Nonpolar LCs. The chemical structures of the LSCE are shown in Figure 1. The polymer backbone is a polymethylhydrosiloxane. The pendent mesogens are 4-(3-butenoxy) benzoic acid-(4-methoxy) phenyl (nonpolar) and 4-(3-butenoxy) benzoic acid-(4cyano) phenyl (polar) attached to the backbone via a hydrosilation reaction. Three samples with different percentages of the two mesogens are synthesized, that is, $50 \mathrm{~mol} \%$ (CN50), $70 \mathrm{~mol} \%$ (CN70), and $100 \mathrm{~mol} \%$ of the CN mesogen (CN100). The fraction of nonpolar mesogen was the complement to complete $100 \mathrm{~mol} \%$ for the mesogens. The networks are chemically cross linked with the bifunctional (hydroquinone diundecyl ether) crosslinker at $7 \mathrm{~mol} \%$ concentration for all the samples. Presumably in the vicinity of $60^{\circ} \mathrm{C}$, the aliphatic 


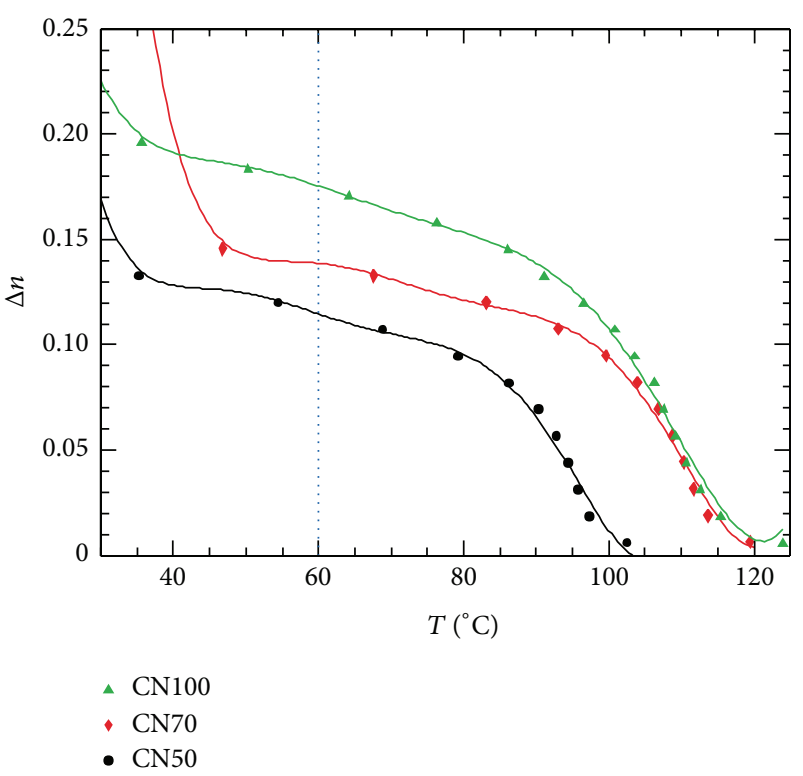

(a)

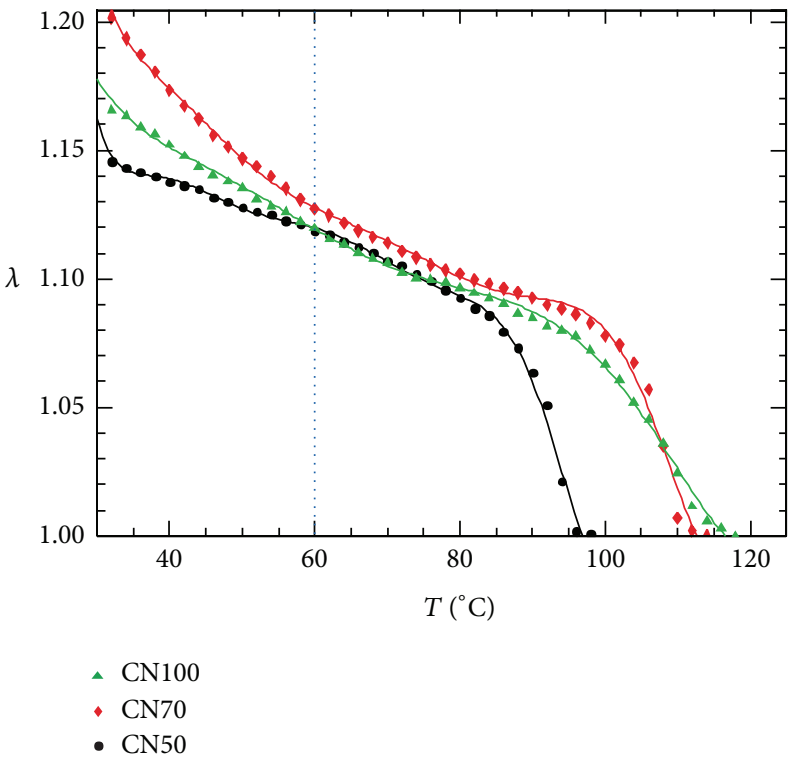

(b)

Figure 5: (a) The orientational order parameter measured by birefringence, $\Delta n$, and (b) spontaneous shape change, $\lambda$, versus $T\left({ }^{\circ} \mathrm{C}\right)$. For $T>40^{\circ} \mathrm{C}, \Delta n$ shows the least order for the shortest mesogens $\bullet$ CN50 and most for $\Delta \mathrm{CN} 100$ that with dimers are the longest. $\bullet \mathrm{CN} 70$, in the middle, has competing monomeric and dimeric tendencies. For spontaneous shape change, CN70 has the largest $\lambda$ and highest $T_{g}$. The solid curves, 6th order polynomial fits to the data, extrapolated to $0^{\circ} \mathrm{C}$ suggest glass transition temperatures, $T_{g} \sim 7-10^{\circ} \mathrm{C}$ for $\mathrm{CN} 50$ and $\mathrm{CN} 100$ and about $10 \mathrm{~K}$ higher for CN70. The dotted line is at $T^{*}=60^{\circ} \mathrm{C}$.

chains of the cross-linker undergo a cis-trans isomerism, or stiffening, making dislocation formation more difficult.

We prepared rectangular LSCEs samples for which the director $\mathbf{n}$ is in the plane of the sample. The sample thickness is $\sim 300 \mu \mathrm{m}$ and area is $\sim 1 \mathrm{~mm} \times 0.5 \mathrm{~mm}$.

The samples were observed with a polarizing microscope (Nikon) equipped with a hot stage (Mettler Toledo FP90 Central Processor) as a temperature controller that can be used simultaneously as a photomonitor as well as for differential scanning calorimetry (DSC). The light source was a $\mathrm{He}-\mathrm{Ne}$ laser. The temperature range, scanned at $0.5 \mathrm{~K} / \mathrm{min}$, was $30^{\circ} \mathrm{C}$ to $130^{\circ} \mathrm{C}$. Birefringence, $\Delta n$, is calculated as in [14].

2.3. Data Analysis. Changes in $\lambda$ and $\Delta n$ are correlated to the microscopic smectic layer spacing, by mapping $\lambda$ and $\Delta n$ to an exceedingly fine temperature scale. The idea is that as the materials are continuous, such a temperature scale is not limited by the precision of the measurements. In other words, there is, in principle, no limit to how fine the temperature scale can be. To optimize accuracy and to obtain a continuous temperature mapping, we fit their nonlinear temperature dependence to a polynomial of order 6 .

A 6th-order polynomial is the least complicated continuous function that fits the $T \leqslant T_{c}$ data well over the whole temperature range while a 4 th-order polynomial fits data in the $T \sim T_{c}$ region less well. Both polynomials show increasingly nonlinear behavior on approaching $T_{g}$, as observed and fitted in CN70 (also in CN50 and CN100 at lower temperatures than in the figures) than a saturationtype behavior implied by a linear continuation from the last data point. This is a normal feature of continuous systems with macroscopic nonlinear properties where information is contained in the data taken together as a whole rather than an exceedingly precise determination of three data points for a linear system. This is fortunate for LCEs which are nonlinear systems for which it is difficult to obtain many data points because of time constraints.

2.4. Spontaneous Length Change, $\lambda$. For spontaneous length changes where strain is induced by temperature, the usual control parameter (temperature in Celsius) is $\varepsilon=\left(T_{c}-\right.$ $T) /\left(T_{c}+273\right)$, such that $\varepsilon \geq 0$ for $T_{c} \geq T$. In Figure 4 , at $T_{c}, \lambda$ and $\Delta n$ decreased slightly faster on heating. We refer to $T_{c}$ as a clearing temperature in the sense that far above $T_{c}, \lambda=1$ and $\Delta n \neq 0$ are constants. A more exact definition for $T_{c}$ does not exist as spontaneous shape change takes place over a broad range of temperatures with exponential decay to the constant values at higher temperatures $[6,7,13]$.

The 6th-order polynomial fits in Figures 4 and 5 exclude the constant valued data above $T_{c}$. For data below $T_{c}$, fits with a minimum in the vicinity of $T \simeq T_{c}$ were selected because above $T_{c}, \lambda$ and $\Delta n$ are nearly constant. In addition, we chose fits where $\Delta n$ and $\lambda$ monotonically increase with decreasing temperature. The minimal polynomial best satisfying these criteria is of order 6 .

Figure 4 compares the three different samples. For example, CN100 approaches $T_{c}^{100}$ more slowly than CN70 and CN50. Indeed, using $T_{c}$ in absolute units as a scaling factor, the temperature dependence of CN70 and CN50 nearly superpose in the vicinity of $\varepsilon=0$. With $\lambda=1$ at $\varepsilon=0$, 
the slopes are $m_{o}^{50}=4.5$ for CN50, $m_{o}^{70}=4.8$ for CN70, and $m_{o}^{100}=1.8$ for $\mathrm{CN100}$. In the intermediate region shown by gray lines in Figure 4, the slopes are $m_{i}^{50}=0.35$, $m_{i}^{70}=0.38$, and $m_{i}^{100}=0.39$ : the slope of CN70 is more similar to CN100 in this temperature interval. The curve $T=$ $T_{i}$ is the lower bound of the intermediate region. Beyond the intermediate regions (also shown by gray lines), CN100 and CN70 show a steeper rise in $\lambda, m_{e}^{100}=0.62$ (offset $=1.03)$ and $m_{e}^{70}=1.05$ (offset $\left.=0.98\right)$ that intersect $m_{i}^{100}$ and $m_{i}^{70}$ at the arrows in the figure flagged for CN70 and $\mathrm{CN100}$. In CN100, this intersection corresponds to a change in slope between two linear regions while in CN70, there is divergence from linearity rendering piecewise linear analysis impractical, motivating the search for the simplest functional form to describe all the data.

The solid curves through $\varepsilon \gtrsim 0$ data are the sixth-order polynomial fits with $\lambda_{0} \simeq 1$ at $\varepsilon=0$ that reflect the influence of a glassy state at $T_{g}$. We discussed $T_{c}$ in some detail because the observation is that whether $\lambda$ diverges or disappears on approaching $T_{g}$ depends on how well the data fit in the vicinity of $T_{c}$. When the data fit well, $\lambda$ and $\Delta n$ diverge; that is, the network becomes "stiffer" with increasing orientational order consistent with observations.

The conclusion is that cross-linking temperature, $T_{c}(K)$, is a good thermodynamic scaling parameter leading to consistent, if not universal, plots for spontaneous shape change in these LSCEs. In particular, as mentioned above, the cyanophenyl in $\mathrm{CN} 100$ requires very high temperatures to activate the enantiomeric mesogenic phases of neat 4cyanophenyl $4^{\prime}$-n-alkoxybenzoate.

2.5. Birefringence, $\Delta n$, versus Spontaneous Length Change, $\lambda$. To make the $\Delta n$ versus $\lambda$ plot (Figure 6), the data are prepared by linear interpolation to create an evenly spaced temperature axis (200 points). As the data do not have massive curvature because the elastomer is both continuous and incompressible, linear interpolation is sufficient. The data are plotted as symbols. For example, the RMS deviation of the $\Delta n$ and $\lambda$ data points from the fitted curves in Figure 5, is better than 2\% superposes the $\Delta n$ versus $\lambda$ data with a 10,000 point expansion of the temperature axis (solid curves for the $\epsilon \geq 0$ data in Figure 4), and fits reasonably well in the vicinity of $T_{c}$.

To include all the data in this study, we fit the interpolated temperature dependence to a polynomial of order 6 . The fitted $\Delta n$ temperature dependence is then replotted with the fitted $\lambda$ as abscissa to obtain Figure 6, showing the correlation between the magnitude of the orientational order parameter with spontaneous shape change.

The degree of orientational order as measured by $\Delta n$ is linear in $\lambda$ as $\lambda \rightarrow 1$ and $\Delta n=0$ when $\lambda=1$. Deviations from linearity are roughly in the vicinity of $T^{*}$, shown by arrows in Figure 6. A summary of these results is

$$
\Delta n=\left\{\begin{array}{lll}
0.75(\lambda-0.94) & \text { for } \lambda<1.05 & \text { CN50 } \\
0.77(\lambda-0.97) & \text { for } \lambda<1.07 & \text { CN70 } \\
1.28(\lambda-0.98) & \text { for } \lambda<1.05 & \text { CN100 }
\end{array} .\right.
$$

A perspective of the transition at $T_{c}$ for an incompressible elastomer is given in [22] where it is argued that this transition

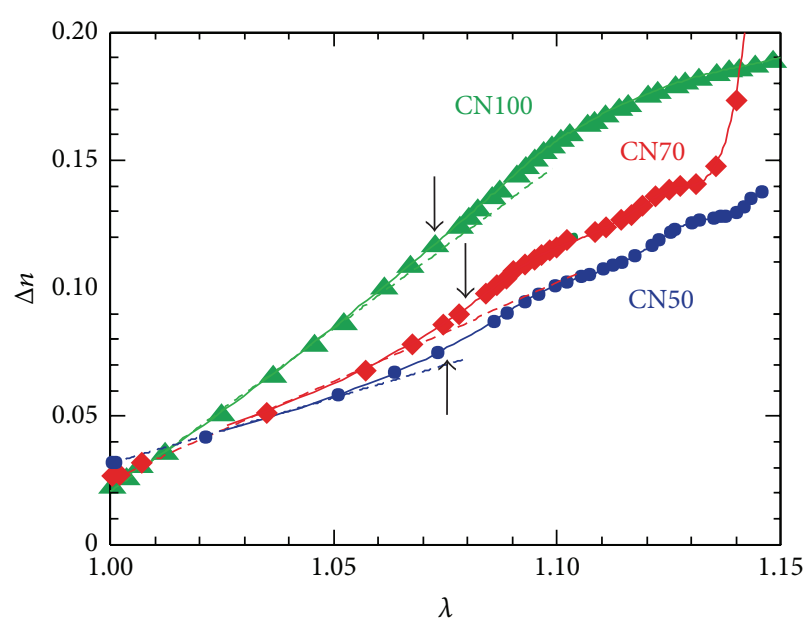

Figure 6: The optical birefringence, $\Delta n$ versus $\lambda$, shows a monotonic increase in orientational order for CN50, CN70, and CN100. The symbols show the $\lambda$ data points from Figure 5 . As $\lambda \rightarrow 1$, $\Delta n$ is linear in $\lambda$ (dashed lines) in all samples up to the value of $\lambda$ at $T^{*}$ (highlighted by arrows) in CN70 and CN100. The deviation from linearity in CN50 is below $T^{*}$. In CN70, $\Delta n$ departs from saturation type behavior at large $\lambda$ in the proximity of an estimated glass transition at $T_{g} \simeq 17-20^{\circ} \mathrm{C}$.

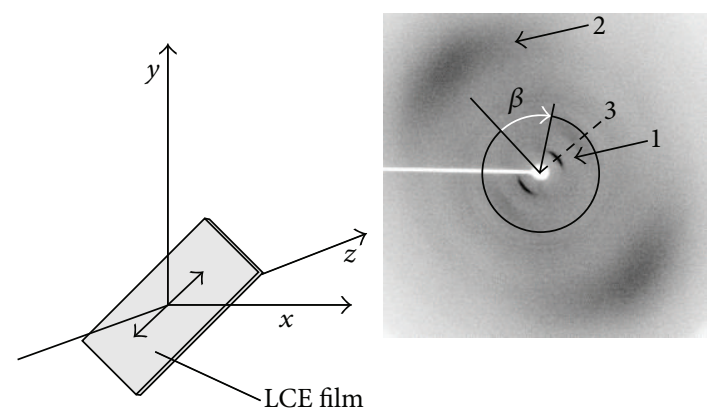

FIGURE 7: Sketch of the experimental setup and the X-ray diffraction pattern of the CN100 LSCE film at $T=60^{\circ} \mathrm{C}$. In the figure, the small angle diffraction peaks (arrow 1 ) are due to smectic clusters, and the wide angle diffraction peaks (arrow 2) are associated with order $\perp \mathbf{n}$.

is an unusual constant-volume first-order phase transition with measurable latent heats when $T_{c}$ is far below the clearing temperature of the LC mesogens cross linked in the elastomer. Taking into account enantiomorphism, a similar perspective for $T_{c}$ far above the clearing temperature could also be applied.

In Figure 6, the change in slope at $T^{*}$ is very small in $\mathrm{CN} 50$, larger in $\mathrm{CN100}$, and largest in CN70. In CN70, the relative increase in $\lambda$ with decreasing temperature becomes smaller and smaller, the closer one is to $T_{g}$. In particular, with increasing the orientational order in $\mathrm{CN} 70$, the change in $\lambda$ becomes smaller at a smaller $\lambda$ than in CN50 and CN100 because it has a higher $T_{g}$. 

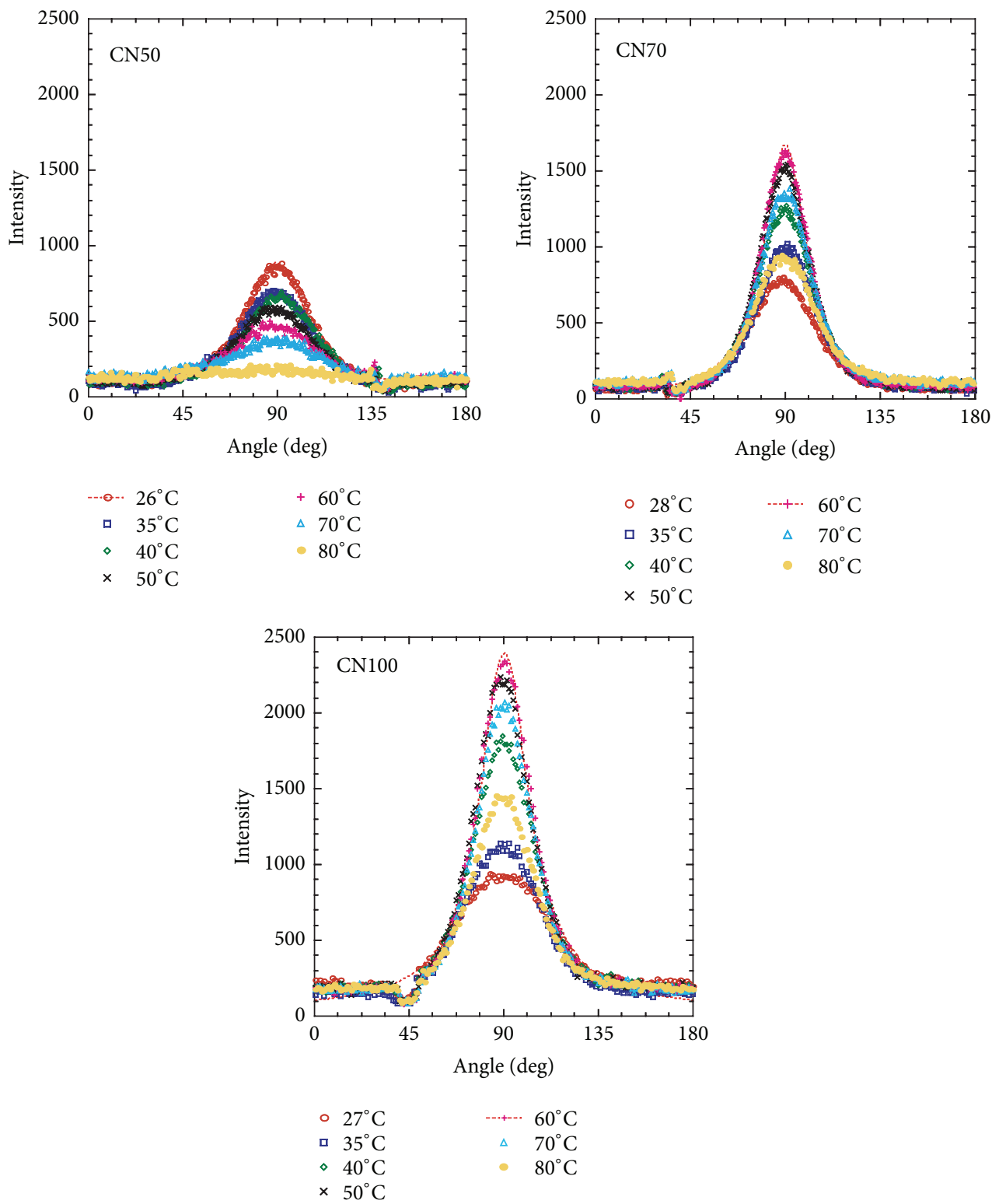

Figure 8: Azimuthal ( $\beta$ in Figure 7) intensity profiles of the small angle peaks at several temperatures for CN50, CN70, and CN100. The intensities are normalized by the background levels due to the polymer backbones. In CN70 and CN100, the peak height (intensity) as well as the peak area are maximum near $T^{*}=60^{\circ} \mathrm{C}$.

\subsection{X-Ray Diffraction}

2.6.1. Structure Factor. To obtain details of the microscopic structure of the elastomers, we performed temperature dependent X-ray diffraction measurements at beamline BL15 of the Kyushu Synchrotron Light Research Center (Japan), where we used $8.00 \mathrm{keV} \mathrm{X-rays} \mathrm{(wavelength} \mathrm{=} 1.550 \AA$ ) filtered by a double crystal monochromator and an image plate system with $4096 \times 8040$ pixels and $50 \mu \mathrm{m}$ resolution. The distance between the sample and the image plate was $180 \mathrm{~mm}$. The imaging geometry is shown in Figure 7.

The temperature-dependent X-ray measurements of the CN50 and CN100 samples covered the temperature range $26^{\circ} \mathrm{C}-80^{\circ} \mathrm{C}$ and $26^{\circ} \mathrm{C}-90^{\circ} \mathrm{C}$ for $\mathrm{CN} 70$. After careful extraction of the background intensity, results for the small angle regime showing smectic information are plotted in Figure 8. While the maximum in the small angle peak intensities at $T=60^{\circ} \mathrm{C}$ is larger in CN100 than in CN70, there are the same number of layers, about 30 , correlated in both samples. As there are never more than 12 layers correlated in $\mathrm{CN} 50$, its peak intensity is smallest.

The best-fit Lorentzian functions for the intensities in Figure 8 are shown with dotted lines. On heating, the degree of smectic order in the CN70 and CN100 samples is maximum in the vicinity of $T^{*} \sim 60^{\circ} \mathrm{C}$, that is, decreases at temperatures both above and below $T^{*}$. 


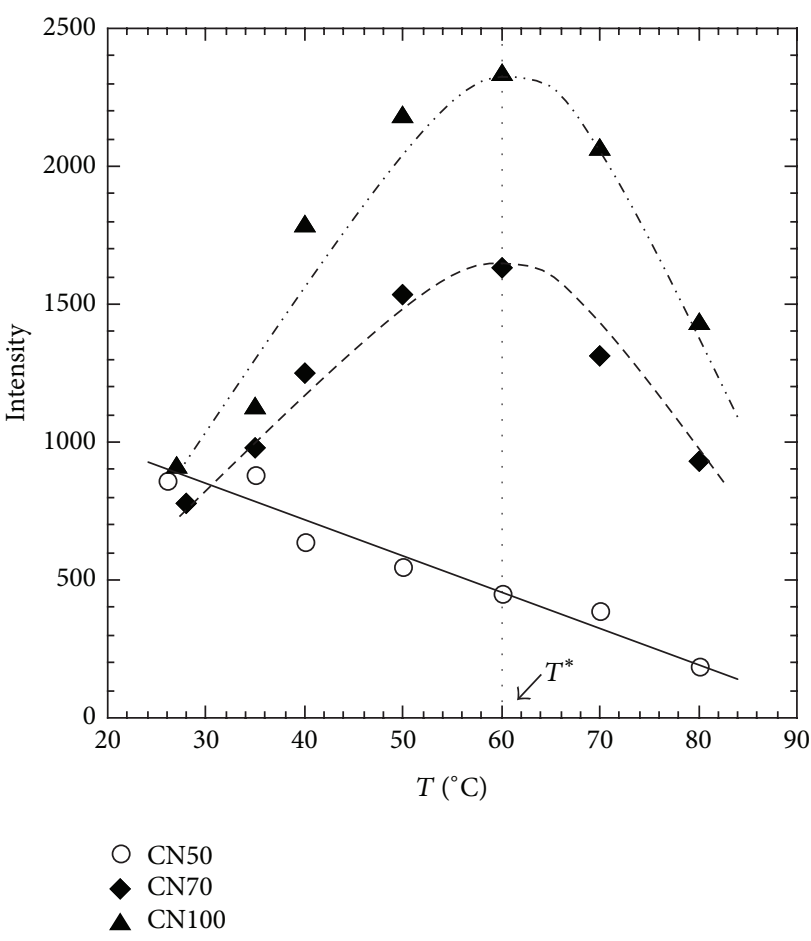

(a)

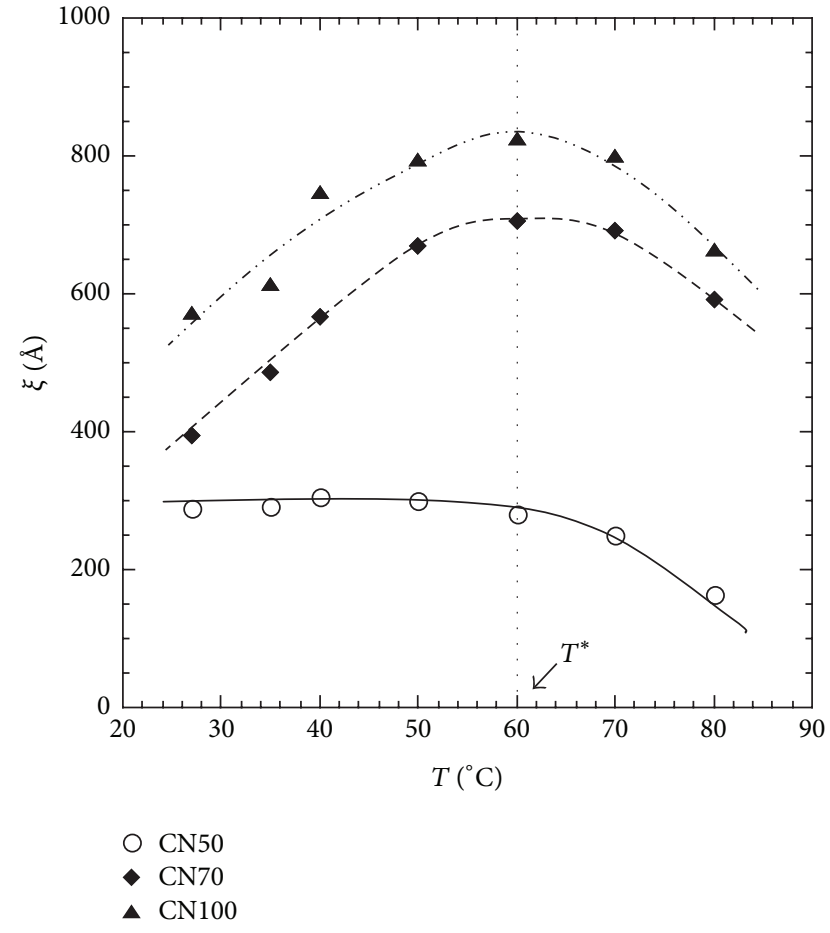

(b)

Figure 9: The small angle peak intensities (a) and $\xi$, the correlation length of the smectic layers calculated from the $2 \theta$ profile, line 3 in Figure 7, are plotted as a function of temperature for the CN50, CN70, and CN100 samples. The solid and dotted lines are guides for the eye. The maximum peak intensities (a) and the maximum correlation length (b) for CN70 and CN100 are observed at $T^{*} \sim 60^{\circ} \mathrm{C}$.

The inverse of the layer spacing, $q_{0}$, is defined by the maximum intensity of $q$ along arrow 1 as indicated by arrow 3, in Figure 7.

2.6.2. Layer Spacing and Correlation to $\lambda$ and $\Delta n$. The temperature dependence of the seven (CN50 and CN100) or eight (CN70) measured values of the layer spacings, $d=2 \pi / q_{0}$ versus $\lambda$, are shown in Figure 10 and versus $\Delta n$ in Figure 11. For a reliable comparison, the data points are connected by lines and the temperature axis expanded to 10,000 points so that the arrows for $60^{\circ} \mathrm{C}$ point exactly to the correct data point, easy to check in CN50 and CN70 but much harder in CN100. With 1000 data points, the temperature scale as defined by $\lambda$ and $\Delta n$ is too coarse for these plots underscoring the key role played by continuity on a macroscopic scale in these materials.

With increasing $\lambda$, the layer spacing initially increases in all samples. Above $T^{*}$, the layer spacing decreases in CN50, hardly changes in CN70, and increases in CN100. As $d$ spacings are made with high precision, the zig-zag appearance for CN70 is interpreted as arising from dislocation unbinding to keep a nearly constant layer spacing in the sample with the largest $\lambda$ and closest to $T_{g}$ (Figures 3 and 10). In contrast, the layer spacing increase in $\mathrm{CN} 100$ is remarkably smooth between $35^{\circ} \mathrm{C}$ and $70^{\circ} \mathrm{C}$ where the layers in the $S_{A}$ clusters are well correlated (Figures 2, 8 and 9).

For $T>T^{*}$, small angle peak intensities associated with smectic order decrease as $T \rightarrow T_{c}$. This is normal.
For example, in CN50, there is no $T^{*}$ and the degree of orientational order saturates at about $60^{\circ} \mathrm{C}$. However, in the cases of CN70 and CN100, there is a maximum in the small angle peak intensities at $T^{*} \simeq 60^{\circ} \mathrm{C}$ where $\lambda$ deviates from saturation-type behavior to one that increases more strongly with decreasing temperature (Figures 6 and 12).

\section{Dislocation Loop Model}

For CN70 and CN100, X-ray diffraction shows a maximum in scattering intensity (Figure $9(\mathrm{a})$ ) and layer correlation length, where $\xi \simeq 700-800 \AA$ (Figure 9(b)) at $T^{*} \sim 60^{\circ} \mathrm{C}$ (Figure 8). As a result, we associate $T^{*}$ with two observations: the onset of nonlinearity in $\lambda$ with increasing $\Delta n$ (Figure 6) and the decrease in layer correlation length, $\xi$, below $T^{*}$ (Figures 3 and 9) and attribute this behavior to dislocation loop formation.

In Figure 12, we plot $\delta \lambda$, the deviation from linearity as a function of temperature below $T^{*}$ for the CN70 and CN100 samples. The dotted lines in the $\delta \lambda-T$ plot are best fits to $\delta \lambda=a\left(T^{*}-T\right) \exp \left(E_{0} / k_{b} T\right)$. Here, $a\left(T^{*}-T\right)$ is related to the number of dislocations in the smectic layers with $a=$ $4 \times 10^{-4} \mathrm{~K}^{-1}$ for both samples. The activation energies are $E_{0}=4.32 \times 10^{-21} \mathrm{~J}$ and $E_{0}=3.79 \times 10^{-21} \mathrm{~J}$ for the CN70 and $\mathrm{CN100}$ samples, respectively. $\delta \lambda$ continuously decreases as the temperature increases, and it does so quite rapidly for the CN70 sample. The suggestion is that conservation of layer spacing requires more dislocations in CN70 for which 


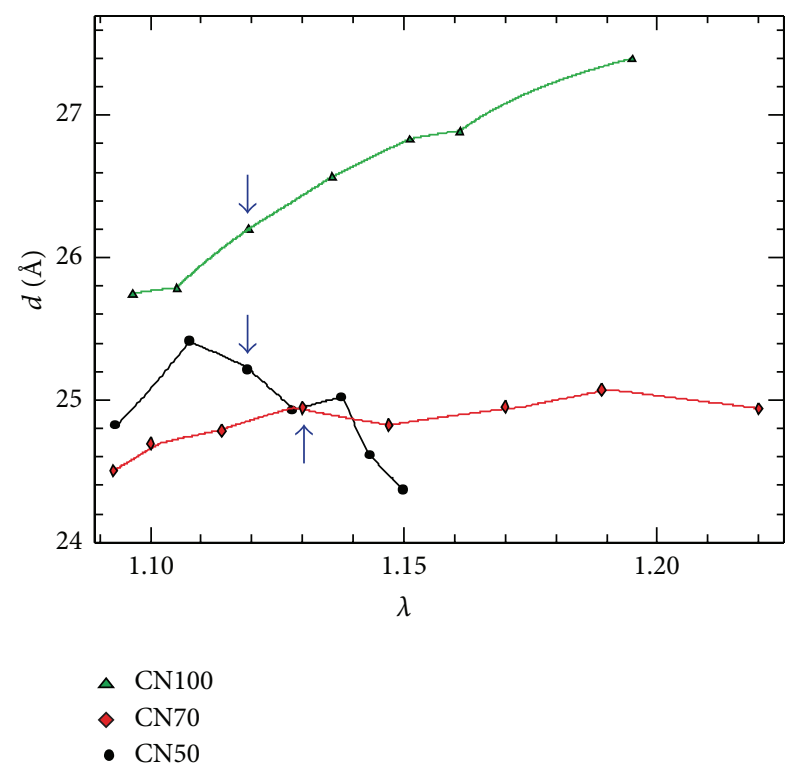

Figure 10: Plot of $d$ versus $\lambda$. The data points are connected by lines. Compared to CN70 and CN50, changes in layer spacings are smooth in CN100. The largest $\lambda$ and the smallest variation in layer spacing are observed for CN70 which has the highest $T_{g}$. Arrows point to $\lambda$ values at $T^{*}=60^{\circ} \mathrm{C}$.

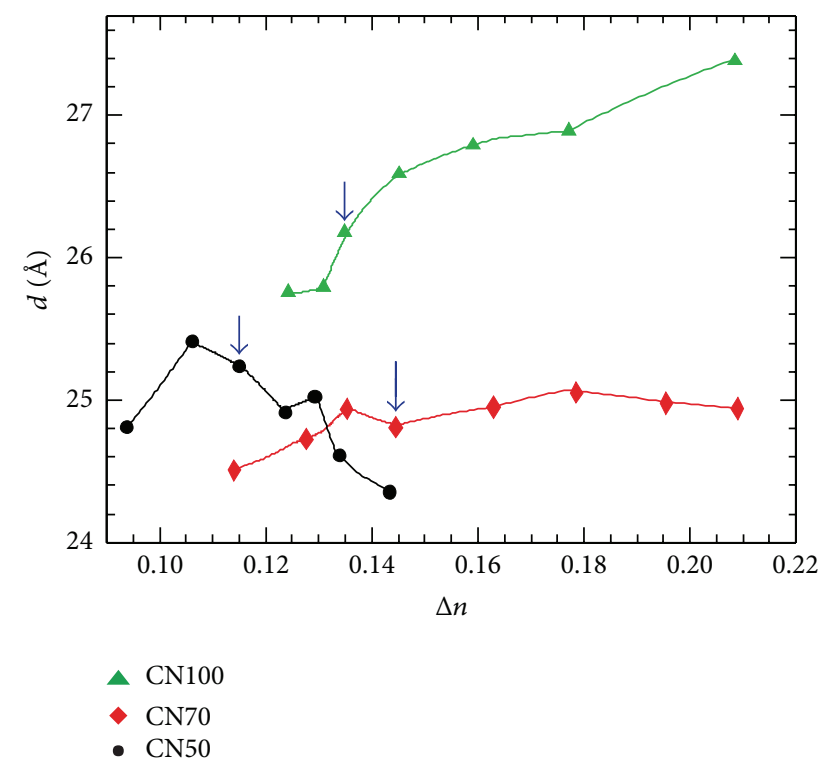

Figure 11: Plot of $d$ versus $\Delta n$. Arrows point to $\Delta n$ values at $T^{*}=$ $60^{\circ} \mathrm{C}$.

$d$ is nearly constant while in $\mathrm{CN} 100, \delta \lambda$ is linear in $T$ as pair formation allows changes in layer spacing with fewer dislocations (Figure 10; see also Figures 3 and 6).

It also suggests that as $T^{*} \sim 60^{\circ} \mathrm{C}$ does not depend on the fraction of CN present; it arises from a molecular transition in the crosslinker present in the same concentration in both CN70 and CN100. For example, cis-trans transitions in the long aliphatic chains of hydroquinone diundecyl ether would change the crosslinker properties from "flexible bifunctional"

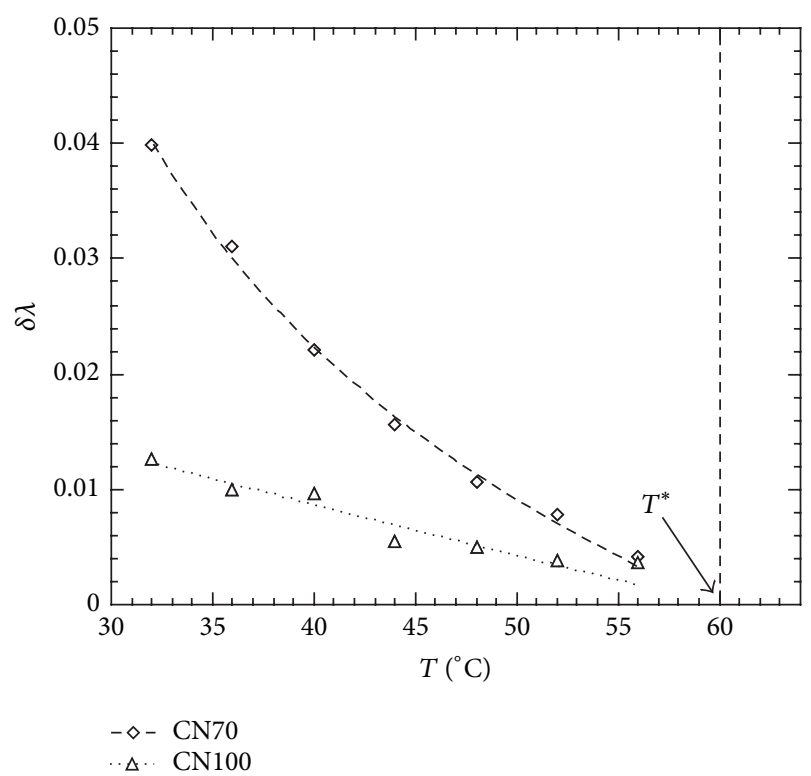

Figure 12: $\delta \lambda$ versus $T$ below $T^{*}$. The dotted lines are best fits to $\delta \lambda=a\left(T^{*}-T\right) \exp \left(E_{0} / k_{b} T\right)$, where $a\left(T^{*}-T\right)$ corresponds to the number of defects and/or smectic domains and $E_{0}$ is the dislocation activation energy.

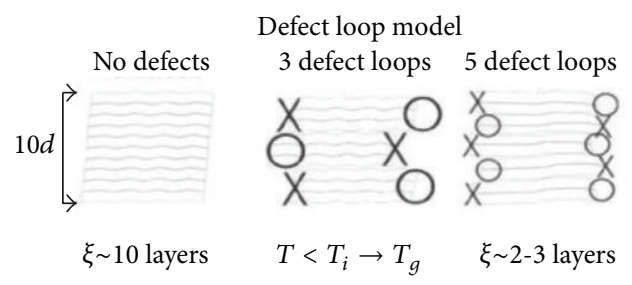

FIGURE 13: Sketch of the dislocation model that conserves layer spacing. $\mathrm{X}$ marks where a loop enters the page and $\bigcirc$ where it emerges from the page. Packets of layers associated with each loop are decorrelated ROM layer packets associated with other loops. As $T \rightarrow T_{g}$, more dislocation loops form leading eventually to no correlated layers in the sample, or, in other words, nematic order.

to "rigid bifunctional" enforcing the appearance of more dislocations that become smaller and smaller as $T \rightarrow T^{*}$ leading to a breakdown in the layer structure.

The idea of a loop is summarized in Figure 13 where packets of correlated layers are held together by a loop however with no correlation between layers in other packets. As more loops form, the number of layers in a packet decreases and layer correlations decrease in the sample leading to the situation shown in Figure 12.

In low-molecular-weight nematic liquid crystals where there are large fluctuations in $\mathbf{n}$ that scatter light, a reentrant nematic is easily observed in the optical microscope with the onset of director fluctuations and sample turbidity. For smectic clusters localized by cross linking in a nematic network, there is no flow and no change in sample turbidity at $T^{*} \sim 60^{\circ} \mathrm{C}$. Rather the effects of increasing dislocation content in LSCEs shows enhancement of orientational order 
and a loss of coherence in the layer structure as more loops form.

\section{Conclusions}

We reported on the spontaneous length change $(\lambda)$, the degree of orientational order measured by birefringence, $\Delta n$, and the X-ray structure factor (Lorentzian) for the transparent nematic network with smectic clusters in three different concentrations of polar and nonpolar mesogens attached to a crosslinked polymer backbone, CN50, CN70, and CN100 corresponding to 50,70 , and $100 \mathrm{~mol} \%$ of the polar mesogen. In the vicinity of $T_{c}$, which depends on concentration of polar moiety, there is a linear relation between $\lambda$ and $\Delta n$ that prevails down to $T^{*} \sim 60^{\circ} \mathrm{C}$. As $T^{*}$ is independent of the concentration of polar moiety, it is attributed to stiffening of the long aliphatic chains of the cross-linker which is present at the same molar concentration in all the samples. In CN50, the maximum in intensity and correlation length of the smectic clusters at $T^{*}$ is maintained at about 10 layers to lower temperatures. In CN70 and CN100, the decrease is from about 30 layers at $T^{*}$ to 10 layers at $T_{g}$. On cooling, the layer spacing in CN50 clusters spacing decreases a little; in CN70, it increases a little and in $\mathrm{CN100}$ it increases more. A dislocation model accounts for the maximum in X-ray scattering at $T^{*}$ as the start of dislocation loop formation leading to a breakdown in layering below $T^{*}$ in CN70 and CN100. More thought, and data are needed to identify the structure (symmetry) of these novel smectic clusters.

\section{Acknowledgments}

This work is partially supported by the Japan-Germany Scientific Cooperative Program of Japan Society for the Promotion of Science (JSPS) and the Deutsche Forschungsgemeinschaft, the Grant for Scientific Research sponsored by JSPS, and the Grant-in-Aid for Scientific Research from the Ministry of Education, Culture, Sports, Science and Technology of Japan (nos. 21340110, 20111003, and 19031023) and Hibah Kompetensi DIKTI. Yusril Yusuf thanks the Research Fellowships of JSPS.

\section{References}

[1] H. Finkelmann, H. J. Kock, and G. Rehage, "Investigations on liquid crystalline polysiloxanes 3. Liquid crystalline elastomers-a new type of liquid crystalline material," Macromolecular Rapid Communications, vol. 2, no. 4, pp. 317-322, 1981.

[2] J. Küpfer and H. Finkelmann, "Nematic liquid single crystal elastomers," Macromolecular Rapid Communications, vol. 12, no. 12, pp. 717-726, 1991.

[3] W. Kaufhold, H. Finkelmann, and H. R. Brand, "Nematic elastomers, 1. Effect of the spacer length on the mechanical coupling between network anisotropy and nematic order," Die Makromolekulare Chemie, vol. 192, no. 11, pp. 2555-2579, 1991.

[4] J. Küpfer and H. Finkelmann, "Liquid crystal elastomers: influence of the orientational distribution of the crosslinks on the phase behaviour and reorientation processes," Macromolecular Chemistry and Physics, vol. 195, no. 4, pp. 1353-1367, 1994.
[5] I. Kundler and H. Finkelmann, "Director reorientation via stripe-domains in nematic elastomers: influence of cross-link density, anisotropy of the network and smectic clusters," Macromolecular Chemistry and Physics, vol. 199, no. 4, pp. 677-686, 1998.

[6] H. R. Brand and K. Kawasaki, "On the macroscopic consequences of frozen order in liquid single crystal elastomers," Macromolecular Rapid Communications, vol. 15, no. 3, pp. 251257, 1994

[7] D. U. Cho, Y. Yusuf, P. E. Cladis, H. R. Brand, H. Finkelmann, and S. Kai, "Trifunctionally cross-linked liquid single crystal elastomers: swelling dynamics and electromechanical effects," Japanese Journal of Applied Physics A, vol. 46, no. 3, pp. 11061113, 2007.

[8] S. Krause, Nematische Hauptkettenelastomere Synthese und Untersuchung der mechanischen Eigenschaften und der Ordnungszustandes, Albert-Ludwigs-Universität Freiburg, 2008.

[9] Y. Yusuf, S. Hashimoto, P. E. Cladis et al., "Main chain liquidcrystalline elastomers: swelling dynamics and electromechanical effects," Molecular Crystals and Liquid Crystals, vol. 508, pp. 367-379, 2009.

[10] P. E. Cladis, P. L. Finn, and J. W. Goodby, in Liquid Crystals and Ordered Fluids, A. C. Griffin and J. F. Johnson, Eds., vol. 4, p. 203, Plenum Press, New York, NY, USA, 1984.

[11] Y. Yusuf, P. E. Cladis, H. R. Brand, H. Finkelmann, and S. Kai, "Hystereses of volume changes in liquid single crystal elastomers swollen with low molecular weight liquid crystal," Chemical Physics Letters, vol. 389, no. 4-6, pp. 443-448, 2004.

[12] Y. Yusuf, Y. Ono, Y. Sumisaki et al., "Swelling dynamics of liquid crystal elastomers swollen with low molecular weight liquid crystals," Physical Review E, vol. 69, no. 2, Article ID 021710, 9 pages, 2004.

[13] D. U. Cho, Y. Yusuf, P. E. Cladis, H. R. Brand, H. Finkelmann, and S. Kai, "Thermo-mechanical properties of tri-functionally crosslinked liquid single crystal elastomers," Chemical Physics Letters, vol. 418, no. 1-3, pp. 217-222, 2006.

[14] Y. Yusuf, P. E. Cladis, H. R. Brand, H. Finkelmann, and S. Kai, "Birefringence measurement of liquid single crystal elastomer swollen with low molecular weight liquid crystal," Chemical Physics Letters, vol. 382, no. 1-2, pp. 198-202, 2003.

[15] Y. Yusuf, N. Minami, S. Yamaguchi et al., "Shape anisotropy and optical birefringence measurements of dry and swollen liquid single crystal elastomers," Journal of the Physical Society of Japan, vol. 76, no. 7, Article ID 073602, 4 pages, 2007.

[16] K. Kawasaki, "Phase dynamics of irregular patterns and ultraslow modes in glass-forming systems," Physica A, vol. 217, no. 1-2, pp. 124-139, 1995.

[17] A. Caillé, Comptes Rendus de l'Académie des Sciences B, vol. 274, p. 891, 1972.

[18] http://mathworld.wolfram.com/LorentzianFunction.html.

[19] D. Demus, J. Goodby, G. W. Gray, H. W. Spiess, and V. Vill, Eds., Physical Properties of Liquid Crystals, Wiley-VCH, New York, NY, USA, 1999.

[20] M. A. Anisimov, P. E. Cladis, E. E. Gorodetskii et al., "Experimental test of a fluctuation-induced first-order phase transition: the nematicsmectic-A transition," Physical Review A, vol. 41, no. 12, pp. 6749-6762, 1990.

[21] P. E. Cladis, in Physical Properties of Liquid Crystals, D. Demus, J. Goodby, G. W. Gray, H. W. Spiess, and V. Vill, Eds., p. 289, Wiley-VCH, New York, NY, USA, 1999.

[22] S. Krause, Y. Yusuf, S. Hashimoto et al., (in preparation). 
[23] P. G. de Gennes, M. Hubert, and R. Kant, "Artificial muscles based on nematic gels," Macromolecular Symposia, vol. 113, no. 1, pp. 39-49, 1997.

[24] M. Hubert, R. Kant, and P. G. de Gennes, "Dynamics and thermodynamics of artificial muscles based on nematic gels," Journal de Physique I, vol. 7, no. 7, pp. 909-919, 1997.

[25] M. Warner and S. Kutter, "Uniaxial and biaxial soft deformations of nematic elastomers," Physical Review E, vol. 65, no. 5, Article ID 051707, 9 pages, 2002. 

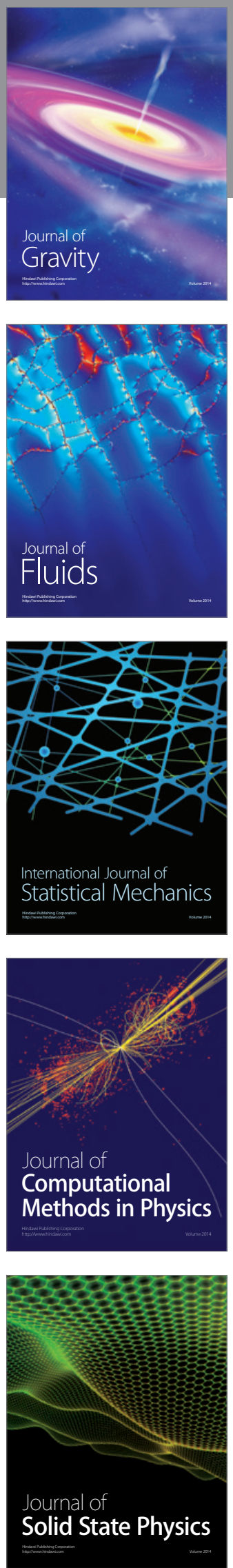

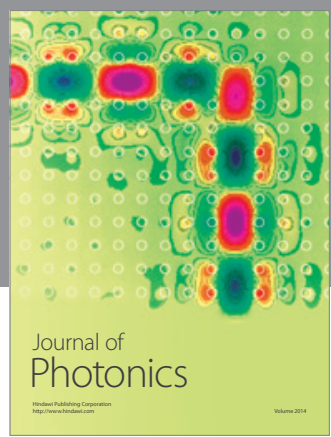

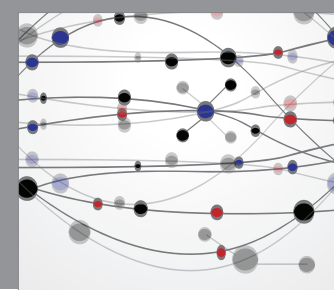

The Scientific World Journal

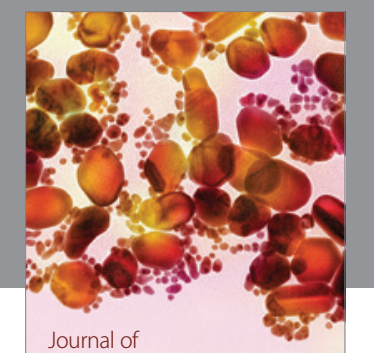

Soft Matter
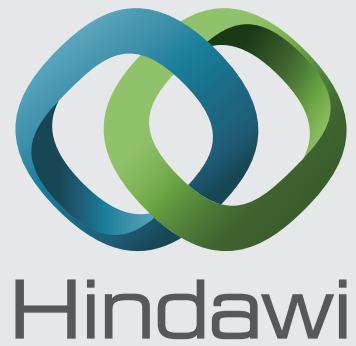

Submit your manuscripts at

http://www.hindawi.com
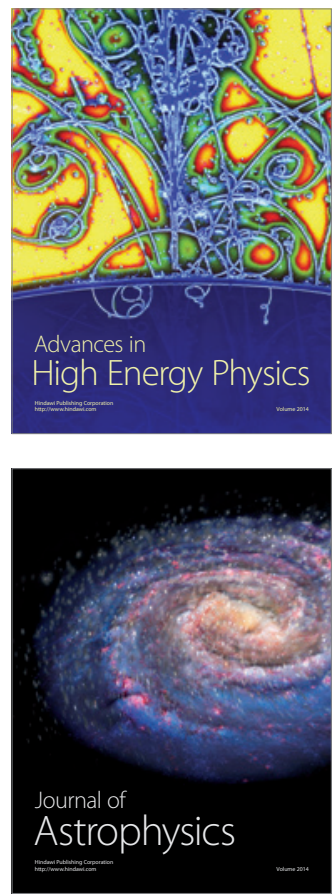
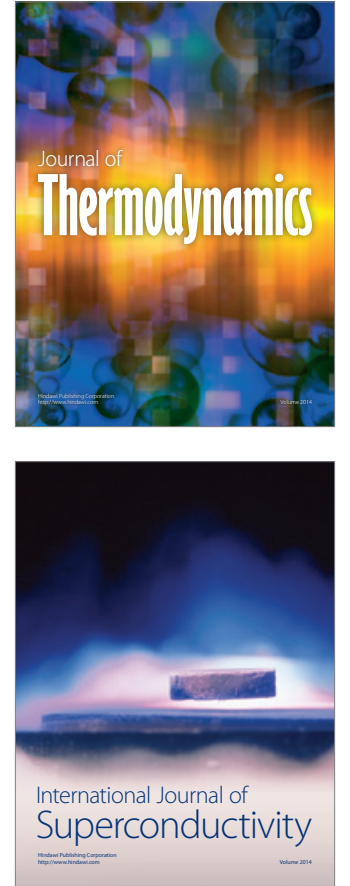
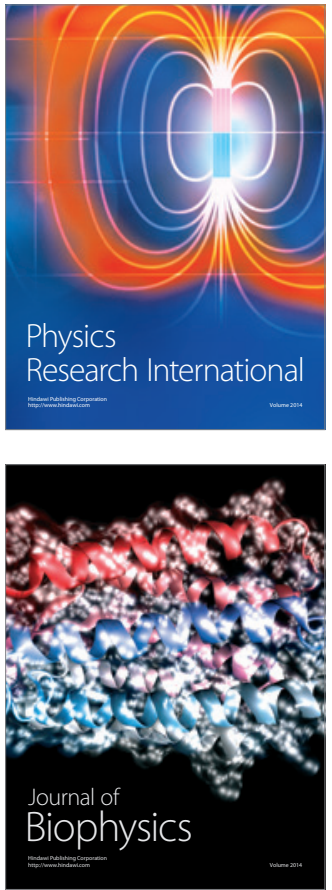
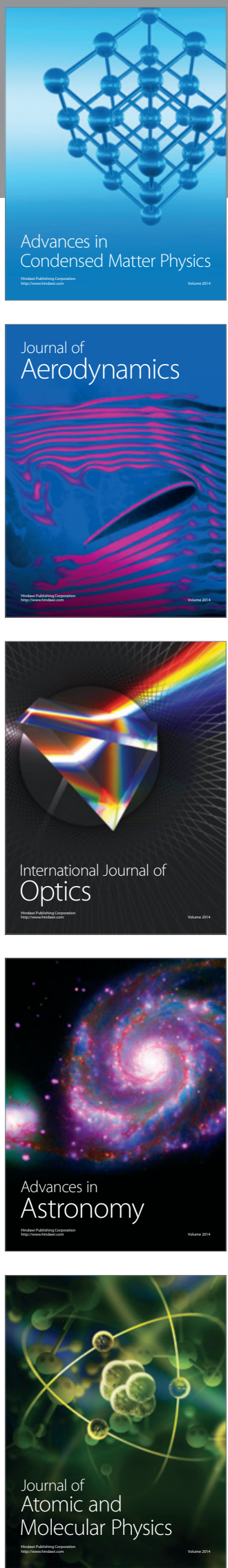